\title{
Uniqueness Theorems in Linear Theory of Microporous Solids
}

\author{
T.G. Gegelia and L. Jentsch
}

\begin{abstract}
This paper deals with uniqueness theorems for external boundary value problems and a contact problem for a system of differential equations of the linear elasticity theory of microporous solids. Here we have derived an asymptotic representation of the solution of this system in a neighbourhood of infinity which has enabled us to prove new uniqueness theorems.
\end{abstract}

Keywords: Linear theory of microporous solids, external boundary value problems, uniqueness theorems

AMS subject classification: Primary $73 \mathrm{C} 15$, secondary $35 \mathrm{Q72}$

\section{Differential equations}

The system of differential equations of the linear elasticity theory of microporous solids is written in the following form (see $[1,3,6,7])$ :

$$
\begin{aligned}
\mu \Delta u+(\lambda+\mu+\eta) g r a d \operatorname{div} u-\eta \operatorname{grad} \theta & =-\varrho f \\
\chi \Delta \theta-\delta \theta+\eta \operatorname{div} u & =0
\end{aligned}
$$

where $u=\left(u_{1}, u_{2}, u_{3}\right)$ is the displacement vector, $\theta$ is the dilatation, $\lambda, \mu$ are the Lamé modules of the solid part of the microporous material, $\varrho$ is the density, and $\eta, \delta, \chi$ are further material constants, whose physical properties are discussed in $[3,6,7]$.

The fundamental equations (1),(2) we can write in the following matrix form:

$$
B\left(\partial_{x}\right) U(x)^{T}+F(x)^{T}=0, \cdots
$$

where

$$
\begin{aligned}
& U=\left(u_{1}, u_{2}, u_{3}, \theta\right), \quad F=\left(\varrho f_{1}, \varrho f_{2}, \varrho f_{3}, 0\right) \\
& U_{i}=u_{i}(i=1,2,3) \text { and } U_{4}=\theta, \quad F_{i}=\varrho f_{i}(i=1,2,3) \text { and } F_{4}=0
\end{aligned}
$$

T.G. Gegelia: Georgian Acad. Sci., Math. Inst., Z. Rukhadze 1, Tbilisi 380093, Georgia L. Jentsch: Techn. Univ. Chemnitz-2wicksu, Dept. Math., PSF 964, D - 09009 Chemnitz. Supported by the Deutsche Forschungsgemeinschaft under grant number Je 166/1-1.

ISSN 0232-2064 / \$2.50 (C) Heldermann Verlag Berlin 


$$
\begin{aligned}
& B\left(\partial_{x}\right)=\left\|B_{i k}\left(\partial_{x}\right)\right\|_{4 \times 4} \\
& B_{i k}\left(\partial_{x}\right)=\delta_{i k} \mu \Delta+\gamma \frac{\partial^{2}}{\partial x_{i} \partial x_{k}}(i, k=1,2,3) \text { and } B_{i 4}\left(\partial_{x}\right)=-\eta \frac{\partial}{\partial x_{i}} \quad(i=1,2,3) \\
& B_{4 k}\left(\partial_{x}\right)=\eta \frac{\partial}{\partial x_{k}}(k=1,2,3) \quad \text { and } \quad B_{44}\left(\partial_{x}\right)=\chi \Delta-\delta
\end{aligned}
$$

$\gamma=\lambda+\mu+\eta, \delta_{i k}$ is the Kronecker symbol. It is assumed that the material constants $\lambda, \mu, \delta, \eta, \chi$ satisfy the conditions (see $[6,7]$ )

$$
\mu, \delta, \chi,\left(\lambda+\frac{2 \mu}{3}+\eta\right) \delta-\eta^{2}>0
$$

The derivation of asymptotic representations mainly rests on the behaviour of the fundamental solution of the considered system.

\section{Fundamental solution}

The fundamental solution of equations (3) can be written in the following form (see $[1,3,6,7] ; i, k=1,2,3)$ :

$$
\begin{aligned}
\Gamma(x) & =\left\|\Gamma_{i k}\right\|_{4 \times 4} \\
\Gamma_{i k}(x) & =-\frac{1}{4 \pi \mu}\left(\frac{\delta_{i k}}{|x|}-\frac{q_{2}}{2 q_{1}} \frac{\partial^{2}|x|}{\partial x_{i} \partial x_{k}}+\frac{\mu \chi \eta^{2}}{q_{1}^{2}} \frac{\partial^{2}}{\partial x_{i} \partial x_{k}} \frac{1-e^{-\frac{\mid \dot{\mid}}{m}}}{|x|}\right) \\
\Gamma_{i 4}(x) & =\frac{1}{4 \pi} \frac{\eta}{q_{1}} \frac{\partial}{\partial x_{i}} \frac{1-e^{-\frac{|s|}{m}}}{|x|} \\
\Gamma_{4 i}(x) & =-\frac{1}{4 \pi} \frac{\eta}{q_{1}} \frac{\partial}{\partial x_{i}} \frac{1-e^{-\frac{|y|}{m}}}{|x|}, \quad \Gamma_{44}(x)=-\frac{e^{-\frac{|y|}{m}}}{4 \pi \chi|x|} \\
q_{1} & =(\lambda+2 \mu+\eta) \delta-\eta^{2}, \quad q_{2}=(\lambda+\mu+\eta) \delta-\eta^{2} \\
m^{2} & =(\lambda+2 \mu+\eta) \frac{\chi}{q_{1}} .
\end{aligned}
$$

By virtue of the inequalities (4) we have

$$
\begin{aligned}
& \lambda+2 \mu+\eta>\frac{\eta^{2}}{\delta}+\frac{4}{3} \mu>0, \quad m^{2}>0, \\
& q_{1}=\frac{4}{3} \mu \delta+\left(\lambda+\frac{2}{3} \mu+\eta\right) \delta-\eta^{2}>\frac{4}{3} \mu \delta>0 .
\end{aligned}
$$

Let us establish the properties of the fundamental matrix $\Gamma=\left\|\Gamma_{i k}\right\|_{4 \times 4}$ in a neighbourhood of infinity. In (5) it was used that

$$
m=+\sqrt{(\lambda+2 \mu+\eta) \frac{\chi}{q_{1}}} .
$$

By virtue of (5) and (6) we have the following estimates:

$$
\begin{aligned}
&\left|\partial^{\alpha} \Gamma_{i k}(x)\right| \leq C|x|^{-1-|\alpha|} \quad(i, k=1,2,3) \\
&\left|\partial^{\alpha} \Gamma_{i 4}(x)\right| \leq C|x|^{-2-|\alpha|} \quad(i=1,2,3) \\
&\left|\partial^{\alpha} \Gamma_{4 k}(x)\right| \leq C|x|^{-2-|\alpha|} \quad(k=1,2,3) \\
&\left|\partial^{\alpha} \Gamma_{44}(x)\right| \leq C|x|^{-n}
\end{aligned}
$$


for some positive number $\mathrm{C}$. Here $\alpha=\left(\alpha_{1}, \alpha_{2}, \alpha_{3}\right)$ is an arbitrary multiindex, $n$ is an arbitrary non-negative integer.

The derivation of the asymptotic representation formula for the solution of the system (3) in a neighbourhood of infinity [2] is based on the Green aud Somigliana formulas [1].

\section{Integral theorems}

Let $\Omega$ be a bounded domain in $\mathbb{R}^{3}$ with a piecewise smooth boundary $\partial \Omega, U=\left(U_{1}, U_{2}\right.$, $\left.U_{3}, U_{4}\right) \in C^{1}(\bar{\Omega}) \cap C^{2}(\Omega), V=\left(V_{1}, V_{2}, V_{3}, V_{4}\right) \in C^{1}(\bar{\Omega}) \cap C^{2}(\Omega), \nu=\left(\nu_{1}, \nu_{2}, \nu_{3}\right)$ the unit normal to $\partial \Omega$ at the point $y$, external with respect to $\Omega$. Then we obtain

$$
\int_{\Omega}\left(V_{i}(z) B_{i k}\left(\partial_{z}\right) U_{k}(z)+2 W(U, V)(z)\right) d z=\int_{\partial \Omega} V_{i}(y) R_{i k}\left(\partial_{y}, \nu\right) U_{k}(y) d_{y} S,
$$

where $W(U, V)$ is a bilinear form:

$$
\begin{aligned}
W(U, V)= & \frac{1}{2}(\lambda+\mu) \sum_{i, j=1}^{3} \frac{\partial U_{i}}{\partial x_{i}} \frac{\partial V_{j}}{\partial x_{j}} \\
& +\frac{\mu}{4} \sum_{i, j=1}^{3}\left(\frac{\partial U_{i}}{\partial x_{j}}+\frac{\partial U_{j}}{\partial x_{i}}\right)\left(\frac{\partial V_{i}}{\partial x_{j}}+\frac{\partial V_{j}}{\partial x_{i}}\right) \\
& -\frac{1}{2} \eta U_{4} \sum_{i=1}^{3} \frac{\partial V_{i}}{\partial x_{i}}-\frac{1}{2} \eta V_{4} \sum_{i=1}^{3} \frac{\partial U_{i}}{\partial x_{i}} \\
& +\frac{1}{2} \delta U_{4} V_{4}+\frac{1}{2} \chi \sum_{i=1}^{3} \frac{\partial U_{4}}{\partial x_{i}} \frac{\partial V_{4}}{\partial x_{i}} \\
R_{i}\left(\partial_{y}, \nu\right)= & \left\|R_{i k}\left(\partial_{y}, \nu\right)\right\|_{4 \times 4} \\
R_{i k}\left(\partial_{y}, \nu\right)= & \delta_{i k \mu} \sum_{j=1}^{3} \nu_{j} \frac{\partial}{\partial y_{j}}+(\lambda+\eta) \nu_{i} \frac{\partial}{\partial y_{k}}+\mu \nu_{k} \frac{\partial}{\partial y_{i}} \quad(i, k=1,2,3) \\
R_{i 4}\left(\partial_{y}, \nu\right)= & -\eta \nu_{i}, \cdots R_{4 i}\left(\partial_{y}, \nu\right)=0 \quad(i=1,2,3) \\
R_{44}\left(\partial_{y}, \nu\right)= & x \sum_{j=1}^{3} \nu_{j} \frac{\partial}{\partial y_{j}} .
\end{aligned}
$$

In the sequel (as in formula (8)) the summation sign $\sum$ will frequently be omitted and the index repetition in the monomial will imply summation from 1 to 4 .

Because of the symmetry $W(U, V)=W(V, U)$, Green's second identity follows from equality (8):

$$
\begin{aligned}
\int_{n}\left(V_{i}(z) B_{i k}\left(\partial_{z}\right) U_{k}(z)-U_{k}(z) B_{k i}\left(\partial_{z}\right) V_{i}(z)\right) d z \\
=\int_{\partial \Omega}\left(V_{i}(y) R_{i k}\left(\partial_{y}, \nu\right) U_{k}(y)-U_{k}(y) R_{k i}\left(\partial_{y}, \nu\right) V_{i}(y)\right) d_{y} S
\end{aligned}
$$

Here summation is taken over the index $i$ and $k$ from 1 to 4 . 
Let $U \in C^{1}(\bar{\Omega}) \cap C^{2}(\Omega)$ and, for all $x \in \Omega, B\left(\partial_{x}\right) U(x) \doteq 0$. Then the Somigliana formula is valid [1], i.e. for all $x \in \Omega$ we have $(j=1,2,3,4)$

$$
U_{j}(x)=\int_{\partial \Omega}\left(U_{i}(y) R_{i k}\left(\partial_{\nu}, \nu\right) \Gamma_{k j}(y-x)-\Gamma_{k j}(y-x) R_{k i}\left(\partial_{y}, \nu\right) U_{i}(y)\right) d_{y} S .
$$

\section{Formulation of the problems}

We consider the following boundary value problems and a general contact problem for the system of differential equations of the elasticity theory of microporous solids. Let $\Omega^{+}$ be a bounded domain in $\mathbb{R}^{3}$,

$$
\Omega^{-}=\mathbb{R}^{3} \backslash \overline{\Omega^{+}}, \quad S=\partial \Omega^{+}=\partial \Omega^{-}
$$

Find in $\Omega^{ \pm}$a solution $U$ of system (3) with one of the boundary conditions below:

- Problems $(1,1)^{ \pm}$: For all $y \in S$ let

$$
U^{+}(y)=\lim _{\Omega^{+}} U(x)=\psi(y), . U^{-}(y)=\lim _{n^{-} \rightarrow x \rightarrow y \in S} U(x)=\psi(y) .
$$

- Problems $(1,2)^{ \pm}$: For all $y \in S$ let

$$
U_{i}^{ \pm}(y)=\psi_{i}(y) \quad(i=1,2,3), \quad \chi\left(\frac{\partial U_{i}}{\partial \nu}\right)^{ \pm}(y)=\psi_{4}(y)
$$

- Problems $(2,1)^{ \pm}$: For all $y \in S$ let

$$
\left(R_{i k}\left(\partial_{y}, \nu\right) U_{k}\right)^{ \pm}(y)=\varphi_{i}(y)(i=1,2,3), \quad U_{4}^{ \pm}(y)=\varphi_{4}(y) .
$$

- Problems $(2,2)^{ \pm}$: For all $y \in S$ let

$$
\left(R\left(\partial_{y}, \nu\right) U\right)^{ \pm}(y)=\varphi(y)
$$

Here $\psi=\left(\psi_{1}, \psi_{2}, \psi_{3}, \psi_{4}\right)$ and $\varphi=\left(\varphi_{1}, \varphi_{2}, \varphi_{3}, \varphi_{4}\right)$ are given functions on $S$. It will be assumed below that a microporous medium, with the material constants $\lambda^{+}, \mu^{+}$, $\eta^{+}, \delta^{+}, \chi^{+}$, occupies domain $\Omega^{+}$and a microporous medium with the material constants $\lambda^{-}, \mu^{-}, \eta^{-}, \delta^{-}, \chi^{-}$occupies domain $\Omega^{-}$. Now let us formulate the general contact problem.

- Problem (3): Find in $\Omega^{+}$and in $\Omega^{-}$a solution $U$ of the system

$$
B^{+}\left(\partial_{x}\right) U(x)=0 \text { for all } x \in \Omega^{+} ; \quad B^{-}\left(\partial_{x}\right) U(x)=0 \text { for all } x \in \Omega^{-}
$$

with the transmission conditions for all $y \in S$

$$
\begin{aligned}
& U^{+}(y)-U^{-}(y)=\psi(y) \\
& \left(R^{+}\left(\partial_{y}, \nu\right) U\right)^{+}(y)-\left(R^{-}\left(\partial_{y}, \nu\right) U\right)^{-}(y)=\varphi(y) .
\end{aligned}
$$

Here $B^{+}, R^{+}$are the operators $B, R$ formed with the material constants $\lambda^{+}, \mu^{+}, \eta^{+}, \delta^{+}$, $\chi^{+}$and $B^{-}, R^{-}$are the operators $B, R$ with the material constants $\lambda^{-}, \mu^{-}, \eta^{-}, \delta^{-}, \chi^{-}$. 


\section{Uniqueness theorem}

A vector $U=\left(U_{1}, U_{2}, U_{3}, U_{4}\right)$, defined in $\Omega^{+}$, we call regular in $\Omega^{+}$if $U \in C^{2}\left(\Omega^{+}\right) \cap$ $C^{1}\left(\overline{\Omega^{+}}\right)$. A vector $U$, defined in $\Omega^{-}$, we call regular in $\Omega^{-}$if $U \in C^{2}\left(\Omega^{-}\right) \cap C^{1}\left(\overline{\Omega^{-}}\right)$and if it admits in a neighbourhood of infinity the estimates

$$
U(x)=O\left(\frac{1}{|x|}\right), \quad \frac{\partial U(x)}{\partial x_{i}}=O\left(\frac{1}{|x|^{2}}\right) .
$$

Let us formulate the uniqueness theorem of a solution of problems $(1,1)^{ \pm},(1,2)^{ \pm},(2,1)^{ \pm}$, $(2,2)^{ \pm},(3)$ (see [1]).

Theorem 1. The problems $(1,1)^{ \pm},(1,2)^{ \pm},(2,1)^{-},(2,2)^{-},(3)$ at best have one regular solution. The difference of two regular solutions of the problem $(2,1)^{+}$or $(2,2)^{+}$is in the set $\operatorname{span}\left(A_{1} ; \ldots A_{6}\right)$, i.e. represented in the form

$$
\sum_{k=1}^{6} c_{k} A_{k} \quad\left(c_{k}=\text { const }\right)
$$

where

$$
A_{k}=\left(\delta_{k 1}, \delta_{k 2}, \delta_{k 3}, 0\right)(k=1,2,3) \text { and } A_{k+3}=\left(\sum_{l=1}^{3} \varepsilon_{k 1 l} x_{l}, \sum_{l=1}^{3} \varepsilon_{k 2 l} x_{l}, \sum_{l=1}^{3} \varepsilon_{k 3 l} x_{l}, 0\right),
$$

$\varepsilon_{k j t}-$ symbol Levi-Civita.

The uniqueness theorems for the external boundary value problems are valid only under some restrictions of the class of solutions at infinity $[1,4,5]$. These restrictions arose naturally from the Green formulas and consist in the requirement that both the solution and its derivatives vanish at infinity (see (11)).

In this paper we will prove the following uniqueness theorem.

Theorem 2. The external boundary value problems $(1,1)^{-},(1,2)^{-},(2,1)^{-},(2,2)^{-}$ and the general contact problem (3) at best have one solution in the class of functions $U$ satisfying the conditions

$$
U \in C^{2}\left(\Omega^{-}\right) \cap C^{1}\left(\overline{\Omega^{-}}\right)
$$

and in a neighbourhood of infinity

$$
U_{i}(x)=o(1) \quad(i=1,2,3,4) .
$$

First we prove the following theorem of the asymptotic representation of a solution of the homogeneous system $B\left(\partial_{x}\right) U(x)=0$ of (3) denoted by (3) .

Theorem 3. Let $\Omega$ be a domain from $\mathbb{R}^{3}$ containing infinity, $U$ defined in $\Omega$ and $U \in C^{2}(\Omega)$. Let in a neighbourhood of $|x|=+\infty$

$$
U_{i}(x)=o\left(|x|^{p+1}\right) \quad(i=1,2,3), \quad \text { and } \quad U_{4}(x)=o\left(|x|^{p}\right)
$$


where $p$ is a non-negative integer. Then in a neighbourhood of $|x|=+\infty$ the following asymptotic representation is valid:

$$
U_{j}(x)=U_{j}^{(0)}(x)+\sum_{|\alpha| \leq p} c_{j}^{(\alpha)} x^{\alpha}
$$

where $U^{(0)}$ is a regular solution of equation (3) in the domain $\Omega$, i.e. $U \in C^{2}(\Omega)$ and, in a neighboorhood of $|x|=+\infty$,

$$
\left|\partial^{\beta} U_{j}^{(0)}(x)\right| \leq \frac{C}{|x|^{1+|\beta|}} \quad(j=1,2,3), \quad\left|\partial^{\beta} U_{4}^{(0)}(x)\right| \leq \frac{C}{|x|^{2+|\beta|}},
$$

where $\beta=\left(\beta_{1}, \beta_{2}, \beta_{3}\right)$ is an arbitrary multiindex.

Theorem 3 can be used, in particular, for Proof of Theorem 2. Indeed, let $U$ be the difference of two solutions of the problem $(p, q)^{-}$or (3) satisfying the conditions (12). Then $(p=0)$ the representation

$$
U_{j}(x)=U_{j}^{(0)}(x)+C_{j}
$$

holds. From (14) and (12) we have $C_{j}=0$ for $j=1,2,3,4$. Therefore $U_{j}(x)=U_{j}^{(0)}(x)$. From Theorem 1 there follows $U_{j}(x)=0$. Thus Theorem 2 is proved

\section{Proof of Theorem 3}

Let $\Omega$ be a domain in $\mathbb{R}^{3}$ containing a neighbourhood of infinity, $x \in \Omega$, and $\tau>1$ chosen such that

$$
x \in B\left(0, \frac{\tau}{8}\right) \quad \text { and } \quad \mathbb{R}^{3} \backslash B\left(0, \frac{\tau}{8}\right) \subset \Omega .
$$

We write the Somigliana formula for the domain $\Omega_{\tau}=B(0, \tau) \cap \Omega$. We will have

$$
\begin{aligned}
U_{j}(x)= & \int_{\partial \Omega}\left(U_{i}(y) R_{i k}\left(\partial_{y}, \nu\right) \Gamma_{k j}(y-x)-\Gamma_{k j}(y-x) R_{k i}\left(\partial_{y}, \nu\right) U_{i}(y)\right) d_{y} S \\
& +\int_{\partial B(0, \tau)}\left(U_{i}(y) R_{i k}\left(\partial_{y}, \nu\right) \Gamma_{k j}(y-x)-\Gamma_{k j}(y-x) R_{k i}\left(\partial_{y}, \nu\right) U_{i}(y)\right) d_{y} S
\end{aligned}
$$

Let

$$
\begin{aligned}
U_{j}^{(0)}(x) & =\int_{\partial \Omega}\left(U_{i}(y) R_{i k}\left(\partial_{y}, \nu\right) \Gamma_{k j}(y-x)-\Gamma_{k j}(y-x) R_{k i}\left(\partial_{y}, \nu\right) U_{i}(y)\right) d_{y} \dot{S} \\
T_{j}(x) & =\int_{\partial B(0, \tau)}\left(U_{i}(y) R_{i k}\left(\partial_{y}, \nu\right) \Gamma_{k j}(y-x)-\Gamma_{k j}(y-x) R_{k i}\left(\partial_{y}, \nu\right) U_{i}(y)\right) d_{y} S .
\end{aligned}
$$

Then

$$
U_{j}(x)=U_{j}^{(0)}(x)+T_{j}(x)
$$

It is not difficult to prove that $U^{(0)}$ is a solution of the equation (3) $)_{0}$. Let us establish the properties of the fundamental matrix

$$
\Gamma_{k j}(y-x)=\Gamma_{j k}(x-y)
$$


We have

$$
\begin{aligned}
B_{l j}\left(\partial_{x}\right) U_{j}^{(0)}(x)= & \int_{\partial \Omega} B_{l j}\left(\partial_{x}\right) U_{i}(y) R_{i k}\left(\partial_{y}, \nu\right) \Gamma_{k j}(y-x) d_{y} S \\
& -\int_{\partial \Omega} B_{l j}\left(\partial_{x}\right) \Gamma_{k j}(y-x) R_{k i}\left(\partial_{y}, \nu\right) U_{i}(y) d_{y} S \\
= & \int_{\partial \Omega} U_{i}(y) R_{i k}\left(\partial_{y}, \nu\right) B_{l j}\left(\partial_{x}\right) \Gamma_{j k}(x-y) d_{y} S \\
& -\int_{\partial \Omega} B_{l j}\left(\partial_{x}\right) \Gamma_{j k}(x-y) R_{k i}\left(\partial_{y}, \nu\right) U_{i}(y) d_{y} S=0 .
\end{aligned}
$$

Now we will prove that $U^{(0)}$ is a regular vector, i.e. in a neighbourhood of $|x|=+\infty$ it admits the estimates (11). Moreover,

$$
\left|\partial^{\alpha} U_{j}^{(0)}(x)\right| \leq \frac{C}{|x|^{1+|\alpha|}} \quad(j=1,2,3) \quad \text { and } \quad\left|\partial^{\alpha} U_{4}^{(0)}(x)\right| \leq \frac{C}{|x|^{2+|\alpha|}}
$$

Indeed, this follows from (7). Let us establish the properties of the integral $T_{j}(x)$ in a neighbourhood of infinity. If $x \neq y$, then $\Gamma_{k j}(y-x)$ is an analytic function. We represent $\Gamma_{k j}(y-x)$ in a neighbourhood of the point $y$ by the Taylor formula:

$$
\begin{aligned}
\Gamma_{k j}(y-x) & =\sum_{|\alpha| \leq p} \frac{(-1)^{|\alpha|} x^{\alpha}}{\alpha !} \partial^{\alpha} \Gamma_{k j}(y)+S_{k j}(x, y) \\
S_{k j}(x ; y) & =\sum_{|\alpha|=p+1} \frac{(-1)^{|\alpha|} x^{\alpha}}{\alpha !} \partial^{\alpha} \Gamma_{k j}(y-\theta x)
\end{aligned}
$$

where $p$ is an arbitrary non-negative integer, $\alpha$ is a multiindex and $0<\theta<1$. First we have to prove that

$$
\partial_{y}^{\beta} S_{k j}(x, y)=\sum_{|\alpha|=p+1} \frac{(-1)^{|\alpha|} x^{\alpha}}{\alpha !} \partial^{\alpha+\beta} \Gamma_{k j}\left(y-\theta_{1} x\right),
$$

where $\beta$ is an arbitrary multiindex and $0<\theta_{1}<1$. The differentiation of (17) gives

$$
\partial_{v}^{\beta} \Gamma_{k j}(y-x)=\sum_{|\alpha| \leq p} \frac{(-1)^{|\alpha|} x^{\alpha}}{\alpha !} \partial^{\beta+\alpha} \Gamma_{k j}(y)+\partial_{y}^{\beta} S_{k j}(\dot{x}, y)
$$

Indeed, using the Taylor formula we can represent $\partial_{y}^{\beta} \Gamma_{k j}(y-x)$ in a neighbourhood of the point $y$ as

$$
\begin{aligned}
& \partial_{y}^{\beta} \Gamma_{k j}(y-x)=\sum_{|\alpha| \leq p} \frac{(-1)^{|\alpha|} x^{\alpha}}{\alpha !} \partial^{j+\alpha} \Gamma_{k j}(y)+S_{k j}^{(1)}(x, y) \\
& S_{k j}^{(1)}(x, y)=\sum_{|\alpha|=p+1} \frac{(-1)^{|\alpha|} x^{\alpha}}{\alpha !} \partial^{\alpha+\beta} \Gamma_{k j}\left(y-\theta_{1} x\right), \quad 0<\theta_{1}<1 .
\end{aligned}
$$


Therefore

$$
\partial_{y}^{\beta} S_{k j}(x, y)=S_{k j}^{(1)}(x, y)
$$

By virtue of (22), (21), (20) and (19) we have (18).

We will estimate $T_{j}(x)$ in a neighbourhood of $|x|=+\infty$. But for all $y \in \partial B(0, \tau)$ $(|y|=\tau)$ and for $x \in B\left(0, \frac{\tau}{8}\right)\left(|x|<\frac{\tau}{8}\right), 0<\theta_{1}<1$, we have

$$
\left|y-\theta_{1} x\right| \geq \frac{7 \tau}{8}=\frac{7|y|}{8} \text {. }
$$

Hence by virtue of (7) we obtain the estimates

$$
\begin{array}{rlr}
\left|\partial^{\alpha+\beta} \Gamma_{k j}\left(y-\theta_{1} x\right)\right| & \leq C_{\alpha, \beta}(x)\left|y-\theta_{1} x\right|^{-1-|\alpha+\beta|} & \\
& \leq C_{\alpha, \beta}(x)|y|^{-2-p-|\beta|} \quad \text { for } k, j=1,2,3 \\
\left|\partial^{\alpha+\beta} \Gamma_{k 4}\left(y-\theta_{1} x\right)\right| & \leq C_{\alpha, \beta}(x)\left|y-\theta_{1} x\right|^{-2-|\alpha+\beta|} & \\
& \leq C_{\alpha, \beta}(x)|y|^{-3-p-|\beta|} \quad \text { for } k=1,2,3 \\
\left|\partial^{\alpha+\beta} \Gamma_{4 k}\left(y-\theta_{1} x\right)\right| & \leq C_{\alpha, \beta}(x)|y|^{-3-p-|\beta|} & \text { for } k=1,2,3 \\
\left|\partial^{\alpha+\beta} \Gamma_{44}\left(y-\theta_{1} x\right)\right| & \leq C_{\alpha, \beta}(x)|y|^{-n-p-|\beta|} &
\end{array}
$$

where $n$ is an arbitrary non-negative integer. Hence we obtain the estimates

$$
\begin{array}{ll}
\left|\partial_{y}^{\beta} S_{k j}(x, y)\right| \leq C_{\alpha, \beta}(x)|y|^{-2-p-|\beta|} & \text { for } k, j=1,2,3 \\
\left|\partial_{y}^{\beta} S_{k j}(x, y)\right| \leq C_{\alpha, \beta}(x)|y|^{-3-p-|\beta|} & \text { for }\left\{\begin{array}{l}
k=4 \text { and } j=1,2,3 \\
k=1,2,3 \text { and } j=4
\end{array}\right. \\
\left|\partial_{y}^{\beta} S_{44}(x, y)\right| \leq C_{\alpha, \beta}(x)|y|^{-n-p-|\beta|} . & \text { - }
\end{array}
$$

The estimates (23) and (9) imply the following ones:

$$
\begin{array}{ll}
\left|R_{i k}\left(\partial_{y}, \nu\right) S_{k j}(x, y)\right| \leq C(x)|y|^{-3-p} & \text { for } i, j=1 ; 2,3 \\
\left|R_{i k}\left(\partial_{y}, \nu\right) S_{k j}(x, y)\right| \leq C(x)|y|^{-4-p} & \text { for } i=4 \text { or } j=4
\end{array}
$$

Let us now turn to the representation (15). We write

$$
\begin{aligned}
T_{j}(x)= & \int_{\partial B(0, \tau)}\left(U_{i}(y) R_{i k}\left(\partial_{y}, \nu\right)\left(\sum_{|\alpha| \leq p} \frac{(-1)^{|\alpha|} x^{\alpha}}{\alpha !} \partial^{\alpha} \Gamma_{k j}(y)+S_{k j}(x, y)\right)\right. \\
& \left.-\left(\sum_{|\alpha| \leq p} \frac{(-1)^{|\alpha|} x^{\alpha}}{\alpha !} \partial^{\alpha} \Gamma_{k j}(y)+S_{k j}(x, y)\right) R_{k i}\left(\partial_{y}, \nu\right) U_{i}(y)\right) d_{y} S \\
= & \sum_{|\alpha| \leq p} C_{j}^{(\alpha)}(\tau) x^{\alpha}+L_{j}(p, \tau, x),
\end{aligned}
$$

where

$$
\begin{aligned}
C_{j}^{(\alpha)}(\tau)= & \frac{(-1)^{|\alpha|}}{\alpha !} \int_{\partial B(0, \tau)}\left(U_{i}(y) R_{i k}\left(\partial_{y}, \nu\right) \partial^{\alpha} \Gamma_{k j}(y)\right. \\
& \left.-\partial^{\alpha} \Gamma_{k j}(y) R_{k i}\left(\partial_{y}, \nu\right) U_{i}(y)\right) d_{y} S \\
L_{j}(p, \tau, x)= & \int_{\partial B(0, \tau)}\left(U_{i}(y) R_{i k}\left(\partial_{y}, \nu\right) S_{k j}(x, y)\right. \\
& \left.-\dot{S}_{k j}(x, y) R_{k i}\left(\partial_{y}, \nu\right) U_{i}(y)\right) d_{y} S
\end{aligned}
$$


We have to show that in the representation (24) the coefficients $C_{j}^{(\alpha)}(\tau)$ are independent of $\tau$. Indeed, let $\tau_{1}>\tau$ and apply Green's formula (10) to the vectors

$$
U=\left(U_{1}, U_{2}, U_{3}, U_{4}\right) \text { and } V=\left(\partial^{\alpha} \Gamma_{1 j}, \partial^{\alpha} \Gamma_{2 j}, \partial^{\alpha} \Gamma_{3 j}, \partial^{\alpha} \Gamma_{4 j}\right)
$$

in the domain $B\left(0, \tau_{1}\right) \backslash \overline{B(0, \tau)}$. When $z \in B\left(0, \tau_{1}\right) \backslash \overline{B(0, \tau)}$, we have

$$
\begin{aligned}
& B_{k i}\left(\partial_{z}\right) V_{i}(z)=B_{k i}\left(\partial_{z}\right) \partial^{\alpha} \Gamma_{i j}(z)=\partial^{\alpha} B_{k i}\left(\partial_{z}\right) \Gamma_{i j}(z)=0 \\
& B_{k i}\left(\partial_{z}\right) U_{i}(z)=0
\end{aligned}
$$

and thus the volume integrals vanish in (10). Therefore we obtain

$$
\begin{aligned}
\int_{\partial B(0, \tau)} & \left(\partial^{\alpha} \Gamma_{i j}(y) R_{i k}\left(\partial_{y}, \nu\right) U_{k}(y)-U_{k}(y) R_{k i}\left(\partial_{y}, \nu\right) \partial^{\alpha} \Gamma_{i j}(y)\right) d_{y} S \\
= & \int_{\partial B\left(0, \tau_{1}\right)}\left(\partial^{\alpha} \Gamma_{i j}(y) R_{i k}\left(\partial_{y}, \nu\right) U_{k}(y)-U_{k}(y) R_{k i}\left(\partial_{y}, \nu\right) \partial^{\alpha} \Gamma_{i j}(y)\right) d_{\nu} S .
\end{aligned}
$$

Here the normals to $\partial B(0, \tau)$ and $\partial B\left(0, \tau_{1}\right)$ are assumed to be external with respect to $B(0, \tau)$ and $B\left(0, \tau_{1}\right)$, respectively. The above equality shows that (see (25)) $C_{0}^{\alpha}(\tau)=$ $C_{s}^{\alpha}\left(\tau_{1}\right)$. Now (24) implies that $L_{j}(p, \tau, x)$ is independent of $\tau$, too.

Now we will prove that if (13) is fulfilled, then

$$
\lim _{\tau \rightarrow \infty} L_{j}(p, \tau, x)=0 .
$$

On account of (27), (24) and (16), we obtain

$$
\dot{U}_{j}(x)=U_{j}^{(0)}(x)+T_{j}(x)=U_{j}^{(0)}(x)+\sum_{|\alpha| \leq p} C_{j}^{(\alpha)} x^{\alpha}
$$

Thus, if we prove (27), we will obtain (28).

Let $w \in C^{\infty}\left(\mathbb{R}^{3}\right)$ be a real function possessing the properties

$$
w(y)=1 \text { for } \frac{1}{2} \leq|y| \leq 2, \quad w(y)=0 \text { for }|y|<\frac{1}{3}, \quad|y|>3 .
$$

Then the function $w^{(\tau)}(y)=w\left(\begin{array}{l}\boldsymbol{k} \\ \tau\end{array}\right)$ possesses the properties:

$$
w^{(\tau)}(y)=1 \text { for } \frac{1}{2} \tau \leq|y| \leq 2 \tau, \quad w^{(\tau)}(y)=0 \text { for }|y|<\frac{\tau}{3}, \quad|y| \geq 3 \tau .
$$

Obviously, $b_{\alpha}=\sup _{\mathbb{R}^{3}}\left|\partial^{\alpha} w(y)\right|<+\infty$ and, therefore;

$$
\left|\partial^{\alpha} w^{(\tau)}(y)\right|=\left|\frac{1}{\tau^{|\alpha|}}\left(\partial^{\alpha} w\right)\left(\frac{y}{\tau}\right)\right| \leq b_{\alpha} \tau^{-|\alpha|}
$$

Introduce the notation

$$
S_{k j}^{(\tau)}(x, y)=w^{(\tau)}(y) S_{k j}(x, y)
$$


Then $S_{k j}^{(r)}$ will have the properties:

$$
\begin{array}{lc}
S_{k j}^{(\tau)}(x, y)=0, \quad R_{i k}\left(\partial_{y}, \nu\right) S_{k j}^{(\tau)}(x, y)=0 & \text { for all } y \in \partial B\left(0, \frac{\tau}{4}\right) \\
S_{k j}^{(\tau)}(x, y)=S_{k j}(x, y), & \\
R_{i k}\left(\partial_{y}, \nu\right) S_{k j}^{(\tau)}(x, y)=R_{i k}\left(\partial_{y}, \nu\right) S_{k j}(x, y) & \text { for all } y \in \partial B(0, \tau) .
\end{array}
$$

Hence by virtue of (26) and (30), we obtain

$$
\begin{aligned}
L_{j}(p, \tau, x) & =\int_{\partial B(0, \tau) \cup \partial B\left(0, \frac{\tau}{\tau}\right)}\left(U_{i}(y) R_{i k}\left(\partial_{y}, \nu\right) S_{k j}^{(\tau)}(x, y)-S_{k j}^{(\tau)}(x, y) R_{k i}\left(\partial_{y}, \nu\right) U_{i}(y)\right) d_{y} S \\
& =\int_{B(0, \tau) \backslash B\left(0, \frac{\tau}{4}\right)}\left(U_{i}(z) B_{i k}\left(\partial_{z}\right) S_{k j}^{(\tau)}(x, z)-S_{k j}^{(\tau)}(x, z) B_{k i}\left(\partial_{z}\right) U_{i}(z)\right) d z \\
& =\int_{B(0, \tau) \backslash B\left(0, \frac{\tau}{4}\right)} U_{i}(z) B_{i k}\left(\partial_{z}\right) S_{k j}^{(\tau)}(x, z) d z .
\end{aligned}
$$

On account of (23), using the above estimate for $\partial^{\alpha} w^{(\tau)}$ (see (13) and (29)) we obtain $U_{i}(z) B_{i k}\left(\partial_{z}\right) S_{k j}^{(\tau)}(x, z)=o\left(\tau^{-3}\right)$ for all $z \in B(0, \tau) \backslash B\left(0, \frac{\tau}{4}\right)$. Thus Theorem 3 is proved

\section{References}

[1] Assaf, M.A. and L. Jentsch: On the elasticity theory of microporous solids. ZAMM 72 (1992), 321 -340 .

[2] Buchukuri, T.V. and T.G. Gegelia: On the uniqueness of solutions of the basic problems of elasticity for infinite domains. Diff. Uravn. 25 (1988), 1556 - 1565.

[3] Eringen, A.C.: Nonlinear theory of micro-elastic solids, I and 11. int. J. Eng. Sci. 2 (1964), 189 203 and $389-404$.

[4] Knops, R.J. and L.E. Payne: Uniqueness Theorems in Linear Elasticity (Springer Tracts in Natura] Philosophy: Vol. 19). Berlin: Springer - Verlag 1971.

[5] Kupradze, V.D., Gegelia, T.G., Basheleishvili, M.O. and T.V. Burchuladze: Three-dimensional problems of the mathematical theory of elasticity and thermoelasticity (North-Holland Series in Applied Mathematics and Mechanics: Vol. 25). Amsterdam: North - Holland Publ. Comp. 1979.

[6] Markov, K.Z.: On the elasticity theory of solids with independent dilatation of particles (in Russian). Theor. Appl. Mech. 5 (1974), 93 - 99.

[7] Markov, K.Z.: On the dilatation theory of elasticity. ZAMM 61 (1981), 349 - 358. 